\title{
Classical Gluon Production Amplitude for Nucleus-Nucleus Collisions: First Saturation Correction in the Projectile
}

\section{Douglas WERTEPNY*}

The Ohio State University

E-mail: wertepny.1@osu.edu

\begin{abstract}
We calculate the classical single-gluon production amplitude in nucleus-nucleus collisions including the first saturation correction in one of the nuclei (the projectile) while keeping multiplerescattering (saturation) corrections to all orders in the other nucleus (the target). In our approximation only two nucleons interact in the projectile nucleus: the single-gluon production amplitude we calculate is order $g^{3}$ and is leading-order in the atomic number of the projectile, while resumming all order-one saturation corrections in the target nucleus. Our result is the first step towards obtaining an analytic expression for the first projectile saturation correction to the gluon production cross section in nucleus-nucleus collisions.
\end{abstract}

XXIII International Workshop on Deep-Inelastic Scattering 27 April - May 12015

Dallas, Texas

*Speaker. 


\section{Introduction}

This proceeding contribution is based on the paper [1].

A complete understanding of heavy-ion collisions and the formation of the quark gluon plasma (QGP) cannot be accomplished without a full understanding of the initial state of the system. This requires knowing the initial distribution of gluons. We would like to calculate the single inclusive gluon production cross-section for heavy-ion collisions at the classical level using perturbative QCD. Since this problem is too difficult at the moment we will instead focus on a simpler problem, heavy-light ion collisions. The goal of the project is to calculate the classical single inclusive gluon production cross-section for heavy-light ion collisions, where we take into account of all of the nucleons in the target and only two of the nucleons in the projectile. Here we present the current status of this calculation, the classical amplitude up to order- $g^{3}$. We start with a review of the proton-nucleus collisions case to describe the main principles of our calculation.

\section{Proton-Nucleus Collisions}

For the high-energy scattering of a projectile proton off a target nucleus, using light-cone coordinates $\left(x^{ \pm}=\left(x^{0} \pm x^{3}\right) / \sqrt{2}\right)$, the projectile (proton) is traveling along the $x^{+}$direction and the target (nucleus) along the $x^{-}$direction. The transverse coordinates are given by $\vec{x}_{\perp}=\left(x^{1}, x^{2}\right)$. The calculation will be preformed in the $A^{+}=0$ light-cone gauge. The gluon propagator is given by

$$
\frac{-i D_{\mu \nu}(l)}{l^{2}+i \varepsilon} \quad \text { where } \quad D_{\mu v}(l)=g_{\mu \nu}-\frac{1}{\eta \cdot l}\left(\eta_{\mu} l_{v}+\eta_{\nu} l_{\mu}\right) .
$$

Due to the high-energy nature of the scattering the projectile sees a Lorentz contracted target and the interaction between the two happens nearly instantaneously compared to the emission time of gluons from the projectile. In the proton-nucleus $(\mathrm{pA})$ collision case the gluon production amplitude, diagrams shown in Fig. 1, has a single nucleon in the projectile and many nucleons in the target. The interactions with the target are instantaneous and, using the saturation/color glass condensate (CGC) framework to model the collision, can be thought of as a shock-wave, represented as a red line in the diagrams. The proton, modeled by a valance quark, can emit a single gluon either before or after the shock wave.
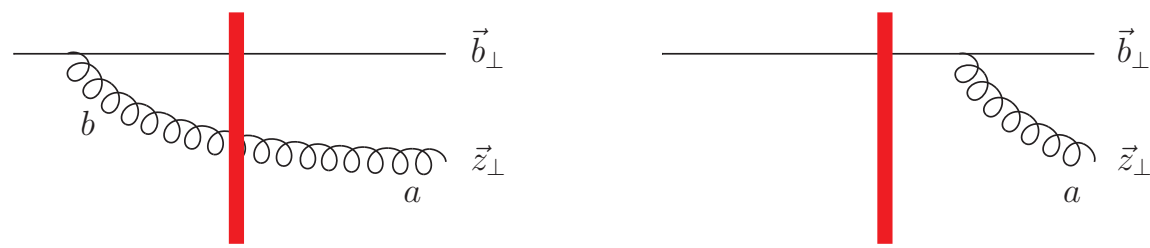

Figure 1: The contributing diagrams to the gluon production amplitude of the pA case. The projectile emits a gluon either before or after the shock-wave, which is represented by a solid red line.

The total amplitude for this process is [3]

$$
M\left(\vec{z}_{\perp}, \vec{b}_{\perp}\right)=\frac{i g}{\pi} \frac{\vec{\varepsilon}_{\perp}^{\lambda *} \cdot\left(\vec{z}_{\perp}-\vec{b}_{\perp}\right)}{\left|\vec{z}_{\perp}-\vec{b}_{\perp}\right|^{2}}\left[U_{\vec{z}_{\perp}}^{a b}-U_{\vec{b}_{\perp}}^{a b}\right]\left(V_{\vec{b}_{\perp}} t^{b}\right)
$$


The shock-wave interactions are represented by Wilson lines in Eq. 2.2, $U_{\vec{x}_{\perp}}^{a b}$ for adjoint and $V_{\vec{x}_{\perp}}$ for fundamental. The Wilson line in the adjoint representation is given by

$$
U_{\vec{x}_{\perp}}^{a b}=\left(\operatorname{Pexp}\left\{i g \int_{-\infty}^{\infty} d x^{+} T^{c} A^{c-}\left(x^{+}, x^{-}=0, x\right)\right\}\right)^{a b}
$$

This represents a gluon traveling through a series of classical gluon fields. In the classical McLerran Venugopalan (MV) [2] model the gluon fields come from nucleon sources in the target. Every scattering comes with a power of $\alpha_{s}^{2} A_{T}^{\frac{1}{3}}(\sim 1$ due to the large number of nucleons in the target), where $A_{T}$ the number of nucleons in the target.

By squaring the amplitude and taking into account the proper normalization constants we arrive at the single inclusive gluon production cross-section,

$$
\begin{aligned}
\frac{d \sigma}{d^{2} k_{T} d y}=\frac{\alpha_{s} C_{F}}{4 \pi^{4}} \int & d^{2} z d^{2} z^{\prime} d^{2} b e^{-i \vec{k}_{\perp} \cdot\left(\vec{z}_{\perp}-\vec{z}_{\perp}^{\prime}\right)} \frac{\vec{z}_{\perp}-\vec{b}_{\perp}}{\left|\vec{z}_{\perp}-\vec{b}_{\perp}\right|^{2}} \cdot \frac{\vec{z}_{\perp}^{\prime}-\vec{b}_{\perp}}{\left|\vec{z}_{\perp}^{\prime}-\vec{b}_{\perp}\right|^{2}} \\
& \times\left[S_{G}\left(\vec{z}_{\perp}, \vec{z}_{\perp}^{\prime}\right)-S_{G}\left(\vec{b}_{\perp}, \vec{z}_{\perp}^{\prime}\right)-S_{G}\left(\vec{z}_{\perp}, \vec{b}_{\perp}\right)+1\right]
\end{aligned}
$$

where

$$
S_{G}\left(\vec{x}_{\perp}, \vec{y}_{\perp}\right)=\frac{1}{N_{C}^{2}-1}\left\langle\operatorname{tr}\left[U_{\vec{x}_{\perp}} U_{\vec{y}_{\perp}}^{\dagger}\right]\right\rangle
$$

is the S-matrix of the gluon dipole.

Now lets examine the power counting. The gluon emission from the quark causes the amplitude squared to gain a factor of $\frac{1}{\alpha_{s}}\left(\alpha_{S}^{2} A_{P}^{\frac{1}{3}}\right)$ where $A_{P}$ is the number of nucleons in the projectile ( $A_{P}=1$ in the proton, but $A_{P}>1$ for a projectile nucleus). The $\frac{1}{\alpha_{s}}$ term is from producing a single classical gluon and $\alpha_{s}^{2} A_{P}^{\frac{1}{3}}(\ll 1)$ is due to the single nucleon in the projectile. The scatterings off the nucleons in the target come with a factor $\left(\alpha_{s}^{2} A_{T}^{\frac{1}{3}}\right)^{N}(\sim 1), N$ being the number of scatterings, and is included in the gluon dipole S-matrix. Overall, the gluon production amplitude in the $\mathrm{pA}$ case (with $N$ rescatterings) is proportional to $\frac{1}{\alpha_{s}}\left(\alpha_{s}^{2} A_{P}^{\frac{1}{3}}\right)\left(\alpha_{s}^{2} A_{T}^{\frac{1}{3}}\right)^{N}$.

\section{Heavy-Light Ion Collisions}

For heavy-light ion collisions the kinematics are the same as in the pA case but the power counting changes. Instead of having a proton as the projectile we have a light ion, meaning we have a large number of nucleons in the projectile, but not as many as in the target, such that $\alpha_{s} \ll$ $\alpha_{S}^{2} A_{P}^{\frac{1}{3}} \lesssim 1$. At leading order the light ion is treated as single nucleon (once again modeled as a single valance quark) which is equivalent to the $\mathrm{pA}$ case. The first saturation correction takes into account two nucleons in the projectile (modeled as two valance quarks). Since $\alpha_{s} \ll \alpha_{s}^{2} A_{P}^{\frac{1}{3}}$ this dominates over the quantum corrections. We still have the $\frac{1}{\alpha_{s}}$ from the single classical gluon production and the power counting of the target nucleons remain the same, $\alpha_{s}^{2} A_{T}^{\frac{1}{3}} \sim 1$. This leads a gluon production amplitude proportional to $\frac{1}{\alpha_{s}}\left(\alpha_{s}^{2} A_{P}^{\frac{1}{3}}\right)^{2}\left(\alpha_{s}^{2} A_{T}^{\frac{1}{3}}\right)^{N}$ for the first order saturation correction. 


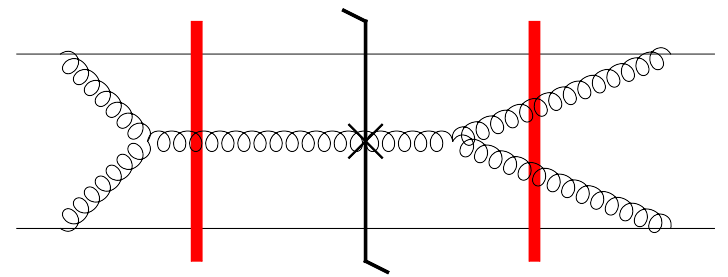

(i)

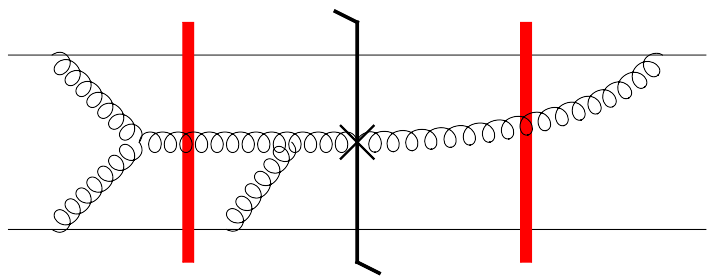

(ii)

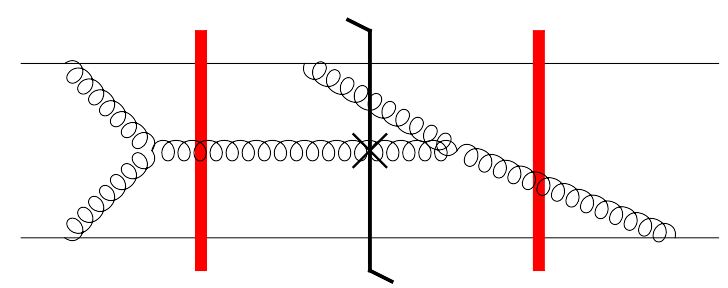

(iii)

Figure 2: The three classes of diagrams: (i) Square of order- $g^{3}$ amplitudes; (ii) Interference between order$g^{5}$ and order- $g$ amplitudes; (iii) Interference between order- $g^{4}$ and order- $g^{2}$ amplitudes.

At this order there are many diagrams which contribute to the amplitude. It is easier to first consider the amplitude squared in which various combinations of diagrams can be combined into single effective diagrams allowing us to simplify the calculation into something more manageable. Fig. 2 shows examples of three main diagram types: (i) square of order- $g^{3}$ amplitudes; (ii) interference between order- $g^{5}$ and order- $g$ amplitudes; (iii) interference between order- $g^{4}$ and order- $g^{2}$ amplitudes. Now we will go through those and show a simplification that can be done with these diagrams to greatly reduce the number of diagrams that must be calculated.
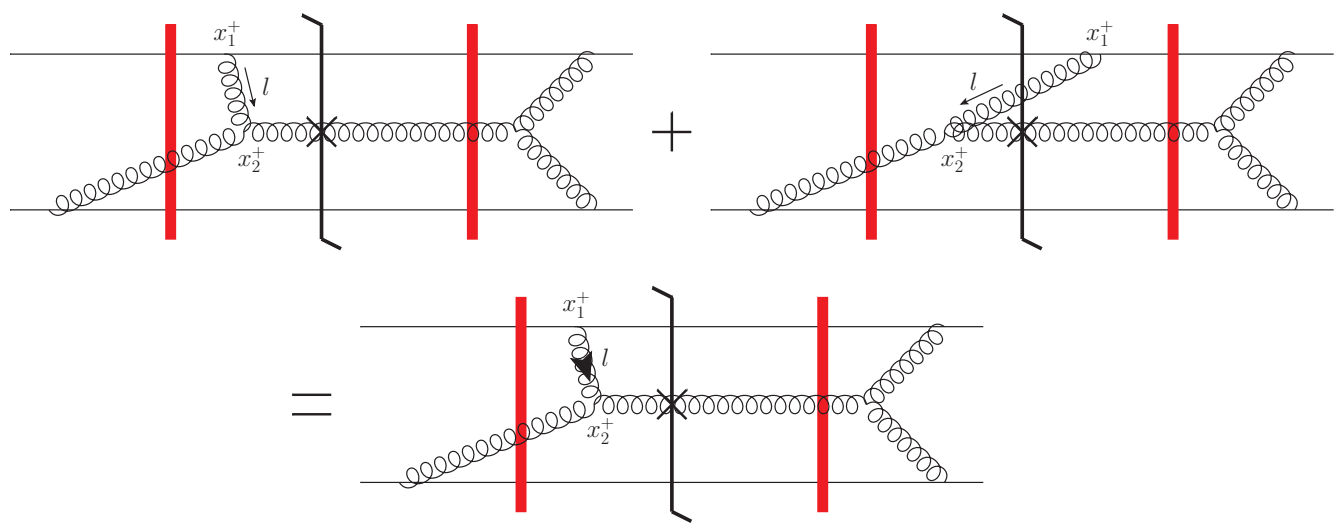

Figure 3: By summing up the diagrams were the gluon is on one side of the cut with the corresponding diagram where said gluon crosses it we get a single diagram with a retarded gluon propagator.

First we can combine all of the order- $g^{2}$ and order- $g^{4}$ diagrams with order- $g^{3}$ and order- $g^{5}$ diagrams through the method shown in Fig. 3. By combining a gluon propagator connected to a quark line on one side of the cut with the diagram where it goes through the cut we can turn the 
gluon propagator into a retarded gluon propagator using

$$
\frac{-i D_{\mu v}(l)}{l^{2}+i \varepsilon}+2 \pi \theta\left(-l^{+}\right) \delta\left(l^{2}\right) D_{\mu v}(l)=\frac{-i D_{\mu v}(l)}{l^{2}+i \varepsilon l^{+}} .
$$

This has the effect of absorbing all of the order- $g^{2}$ and order- $g^{4}$ diagrams into order- $g^{3}$ and order- $g^{5}$ diagrams where all of the gluon propagators have been replaced with retarded propagators. There are other tricks that allow further simplification which are explained in more detail in [1].

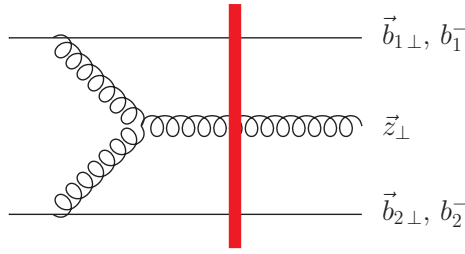

$\mathbf{A}_{1}$

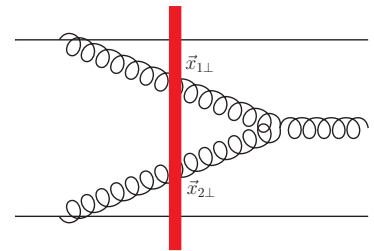

$\mathbf{A}_{2}$

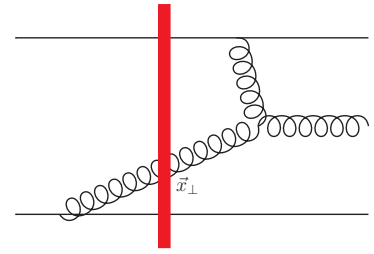

$\mathbf{A}_{3}$

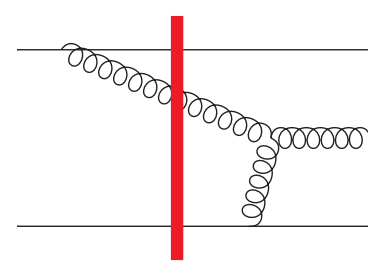

$\mathbf{A}_{4}$

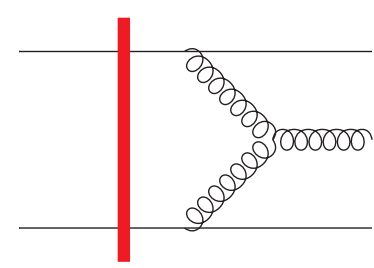

$\mathrm{A}_{5}$

Figure 4: A-diagrams have a single 3-gluon vertex with gluons connecting to both quarks. All gluons are retarded and the time flows to the right.

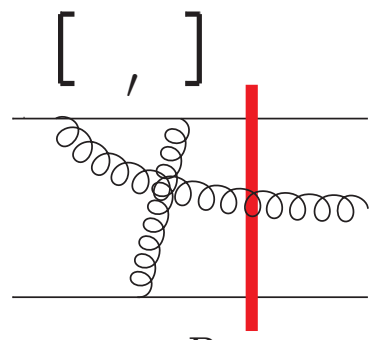

$\mathrm{B}_{2}$

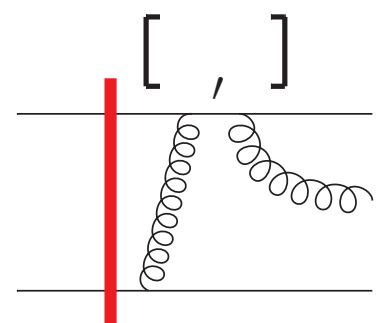

$\mathbf{B}_{9}$

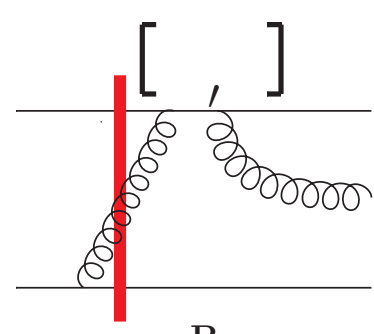

$\mathbf{B}_{11}$

Figure 5: B-diagrams consist of a single gluon emission with one gluon traveling between the quarks. Notice the color factor associated with the top quark has been replaced with a commutator, represented by a bracket, $[$,$] . The gluons connecting the quarks are retarded with the momentum flowing to the top quark$ line.

As a result we end up with only a few diagrams. The order- $g^{3}$ gluon production diagrams are divided up into 5 different types (A, B, C, D and E): Type A involves a process with a single 3-gluon vertex with gluons connecting it to both quarks, Fig. 4; Type B consists of an emission of a single gluon with another gluon traveling between the quarks where the color factor of the upper quark has been replaced with a commutator, Fig. 5; Type $\mathrm{C}$ are the same as type $\mathrm{B}$ with the valance quarks interchanged; Type D involves only one valence quark at the amplitude level (this implies that only the other valance quark is involved on the other side of the cut), Fig. 6; Type E are obtained from 

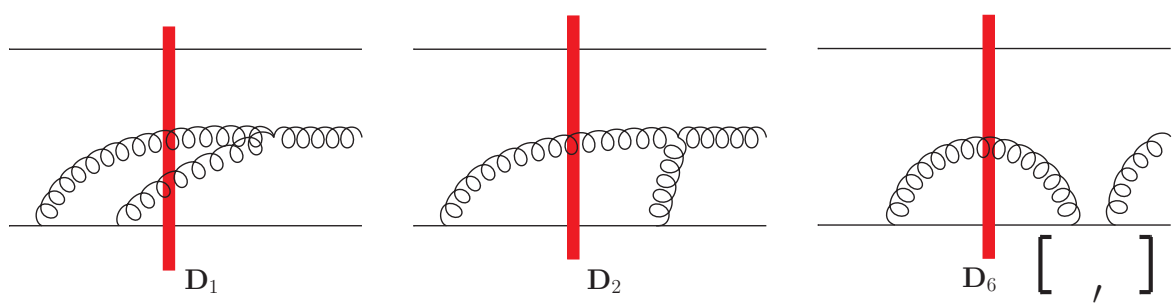

Figure 6: D-diagrams involve only one quark at the amplitude level (this implies that only the other quark is involved on the other side of the cut). All gluons are retarded with momentum flowing to the right.

type D by interchanging valence quarks. The order- $g^{5}$ diagrams are not included here and are left for future work. With these diagrams in hand we calculated the amplitude in transverse coordinate space and came up with a relatively compact result for the A, B and C-diagrams,

$$
\begin{aligned}
& \sum A_{i}+\sum B_{i}+\sum C_{i} \\
& =-\frac{g^{3}}{4 \pi^{4}} \int d^{2} x_{1} d^{2} x_{2} \delta\left[\left(\vec{z}_{\perp}-\vec{x}_{1 \perp}\right) \times\left(\vec{z}_{\perp}-\vec{x}_{2 \perp}\right)\right]\left[\frac{\vec{\varepsilon}_{\perp}^{\lambda *} \cdot\left(\vec{x}_{2 \perp}-\vec{x}_{1 \perp}\right)}{\left|\vec{x}_{2 \perp}-\vec{x}_{1 \perp}\right|^{2}} \frac{\vec{x}_{1 \perp}-\vec{b}_{1 \perp}}{\left|\vec{x}_{1 \perp}-\vec{b}_{1 \perp}\right|^{2}} \cdot \frac{\vec{x}_{2 \perp}-\vec{b}_{2 \perp}}{\left|\vec{x}_{2 \perp}-\vec{b}_{2 \perp}\right|^{2}}\right. \\
& \left.-\frac{\vec{\varepsilon}_{\perp}^{\lambda *} \cdot\left(\vec{x}_{1 \perp}-\vec{b}_{1 \perp}\right)}{\left|\vec{x}_{1 \perp}-\vec{b}_{1 \perp}\right|^{2}} \frac{\vec{z}_{\perp}-\vec{x}_{1 \perp}}{\left|\vec{z}_{\perp}-\vec{x}_{1 \perp}\right|^{2}} \cdot \frac{\vec{x}_{2 \perp}-\vec{b}_{2 \perp}}{\left|\vec{x}_{2 \perp}-\vec{b}_{2 \perp}\right|^{2}}+\frac{\vec{\varepsilon}_{\perp}^{\lambda *} \cdot\left(\vec{x}_{2 \perp}-\vec{b}_{2 \perp}\right)}{\left|\vec{x}_{2 \perp}-\vec{b}_{2 \perp}\right|^{2}} \frac{\vec{x}_{1 \perp}-\vec{b}_{1 \perp}}{\left|\vec{x}_{1 \perp}-\vec{b}_{1 \perp}\right|^{2}} \cdot \frac{\vec{z}_{\perp}-\vec{x}_{2 \perp}}{\left|\vec{z}_{\perp}-\vec{x}_{2 \perp}\right|^{2}}\right] \\
& \times f^{a b c}\left[U_{\vec{x}_{1 \perp}}^{b d}-U_{\vec{b}_{1 \perp}}^{b d}\right]\left[U_{\vec{x}_{2 \perp}}^{c e}-U_{\vec{b}_{2 \perp}}^{c e}\right]\left(V_{\vec{b}_{1 \perp}} t^{d}\right)_{1}\left(V_{\vec{b}_{2 \perp}} t^{e}\right)_{2} \\
& +\frac{i g^{3}}{4 \pi^{3}} f^{a b c}\left(V_{\vec{b}_{1 \perp}} t^{d}\right)_{1}\left(V_{\vec{b}_{2 \perp}} t^{e}\right)_{2} \int d^{2} x\left[U _ { \vec { b } _ { 1 \perp } } ^ { b d } ( U _ { \vec { x } _ { \perp } } ^ { c e } - U _ { \vec { b } _ { 2 \perp } } ^ { c e } ) \left(\frac{\vec{\varepsilon}_{\perp}^{\lambda *} \cdot\left(\vec{z}_{\perp}-\vec{x}_{\perp}\right)}{\left|\vec{z}_{\perp}-\vec{x}_{\perp}\right|^{2}} \frac{\vec{x}_{\perp}-\vec{b}_{1 \perp}}{\left|\vec{x}_{\perp}-\vec{b}_{1 \perp}\right|^{2}} \cdot \frac{\vec{x}_{\perp}-\vec{b}_{2 \perp}}{\left|\vec{x}_{\perp}-\vec{b}_{2 \perp}\right|^{2}}\right.\right. \\
& \left.-\frac{\vec{\varepsilon}_{\perp}^{\lambda *} \cdot\left(\vec{z}_{\perp}-\vec{b}_{1 \perp}\right)}{\left|\vec{z}_{\perp}-\vec{b}_{1 \perp}\right|^{2}} \frac{\vec{z}_{\perp}-\vec{x}_{\perp}}{\left|\vec{z}_{\perp}-\vec{x}_{\perp}\right|^{2}} \cdot \frac{\vec{x}_{\perp}-\vec{b}_{2 \perp}}{\left|\vec{x}_{\perp}-\vec{b}_{2 \perp}\right|^{2}}-\frac{\vec{\varepsilon}_{\perp}^{\lambda *} \cdot\left(\vec{z}_{\perp}-\vec{b}_{1 \perp}\right)}{\left|\vec{z}_{\perp}-\vec{b}_{1 \perp}\right|^{2}} \frac{\vec{x}_{\perp}-\vec{b}_{1 \perp}}{\left|\vec{x}_{\perp}-\vec{b}_{1 \perp}\right|^{2}} \cdot \frac{\vec{x}_{\perp}-\vec{b}_{2 \perp}}{\left|\vec{x}_{\perp}-\vec{b}_{2 \perp}\right|^{2}}\right) \\
& -\left(U_{\vec{x}_{\perp}}^{b d}-U_{\vec{b}_{1 \perp}}^{b d}\right) U_{\vec{b}_{2 \perp}}^{c e}\left(\frac{\vec{\varepsilon}_{\perp}^{\lambda *} \cdot\left(\vec{z}_{\perp}-\vec{x}_{\perp}\right)}{\left|\vec{z}_{\perp}-\vec{x}_{\perp}\right|^{2}} \frac{\vec{x}_{\perp}-\vec{b}_{1 \perp}}{\left|\vec{x}_{\perp}-\vec{b}_{1 \perp}\right|^{2}} \cdot \frac{\vec{x}_{\perp}-\vec{b}_{2 \perp}}{\left|\vec{x}_{\perp}-\vec{b}_{2 \perp}\right|^{2}}-\frac{\vec{\varepsilon}_{\perp}^{\lambda *} \cdot\left(\vec{z}_{\perp}-\vec{b}_{2 \perp}\right)}{\left|\vec{z}_{\perp}-\vec{b}_{2 \perp}\right|^{2}} \frac{\vec{z}_{\perp}-\vec{x}_{\perp}}{\left|\vec{z}_{\perp}-\vec{x}_{\perp}\right|^{2}} \cdot \frac{\vec{x}_{\perp}-\vec{b}_{1 \perp}}{\left|\vec{x}_{\perp}-\vec{b}_{1 \perp}\right|^{2}}\right. \\
& \left.\left.-\frac{\vec{\varepsilon}_{\perp}^{\lambda *} \cdot\left(\vec{z}_{\perp}-\vec{b}_{2 \perp}\right)}{\left|\vec{z}_{\perp}-\vec{b}_{2 \perp}\right|^{2}} \frac{\vec{x}_{\perp}-\vec{b}_{1 \perp}}{\left|\vec{x}_{\perp}-\vec{b}_{1 \perp}\right|^{2}} \cdot \frac{\vec{x}_{\perp}-\vec{b}_{2 \perp}}{\left|\vec{x}_{\perp}-\vec{b}_{2 \perp}\right|^{2}}\right)\right] \\
& -\frac{i g^{3}}{4 \pi^{2}} f^{a b c}\left(V_{\vec{b}_{1 \perp}} t^{d}\right)_{1}\left(V_{\vec{b}_{2 \perp}} t^{e}\right)_{2} \\
& \times\left[\left(U_{\vec{z}_{\perp}}^{b d}-U_{\vec{b}_{1 \perp}}^{b d}\right) U_{\vec{b}_{2 \perp}}^{c e} \frac{\vec{\varepsilon}_{\perp}^{\lambda *} \cdot\left(\vec{z}_{\perp}-\vec{b}_{1 \perp}\right)}{\left|\vec{z}_{\perp}-\vec{b}_{1 \perp}\right|^{2}} \ln \frac{1}{\left|\vec{z}_{\perp}-\vec{b}_{2 \perp}\right| \Lambda}-U_{\vec{b}_{1 \perp}}^{b d}\left(U_{\vec{z}_{\perp}}^{c e}-U_{\vec{b}_{2 \perp}}^{c e}\right) \frac{\vec{\varepsilon}_{\perp}^{\lambda *} \cdot\left(\vec{z}_{\perp}-\vec{b}_{2 \perp}\right)}{\left|\vec{z}_{\perp}-\vec{b}_{2 \perp}\right|^{2}} \ln \frac{1}{\left|\vec{z}_{\perp}-\vec{b}_{1 \perp}\right| \Lambda}\right] \\
& -\frac{i g^{3}}{4 \pi^{3}} \int d^{2} x\left[U_{x_{\perp}}^{a b}-U_{z_{\perp}}^{a b}\right] f^{b d e}\left(V_{\vec{b}_{1 \perp}} t^{d}\right)_{1}\left(V_{\vec{b}_{2 \perp}} t^{e}\right)_{2} \\
& \times \frac{\vec{\varepsilon}_{\perp}^{\lambda *} \cdot\left(\vec{z}_{\perp}-\vec{x}_{\perp}\right)}{\left|\vec{z}_{\perp}-\vec{x}_{\perp}\right|^{2}} \frac{\vec{x}_{\perp}-\vec{b}_{1 \perp}}{\left|\vec{x}_{\perp}-\vec{b}_{1 \perp}\right|^{2}} \cdot \frac{\vec{x}_{\perp}-\vec{b}_{2 \perp}}{\left|\vec{x}_{\perp}-\vec{b}_{2 \perp}\right|^{2}} \operatorname{Sign}\left(b_{2}^{-}-b_{1}^{-}\right),
\end{aligned}
$$


and for the D-diagrams,

$$
\begin{aligned}
& \sum D_{i}=-\frac{g^{3}}{8 \pi^{4}} \int d^{2} x_{1} d^{2} x_{2} \delta\left[\left(\vec{z}_{\perp}-\vec{x}_{1 \perp}\right) \times\left(\vec{z}_{\perp}-\vec{x}_{2 \perp}\right)\right]\left[\frac{\vec{\varepsilon}_{\perp}^{\lambda *} \cdot\left(\vec{x}_{2 \perp}-\vec{x}_{1 \perp}\right)}{\left|\vec{x}_{2 \perp}-\vec{x}_{1 \perp}\right|^{2}} \frac{\vec{x}_{1 \perp}-\vec{b}_{2 \perp}}{\left|\vec{x}_{1 \perp}-\vec{b}_{2 \perp}\right|^{2}} \cdot \frac{\vec{x}_{2 \perp}-\vec{b}_{2 \perp}}{\left|\vec{x}_{2 \perp}-\vec{b}_{2 \perp}\right|^{2}}\right. \\
& \left.-\frac{\vec{\varepsilon}_{\perp}^{\lambda *} \cdot\left(\vec{x}_{1 \perp}-\vec{b}_{2 \perp}\right)}{\left|\vec{x}_{1 \perp}-\vec{b}_{2 \perp}\right|^{2}} \frac{\vec{z}_{\perp}-\vec{x}_{1 \perp}}{\left|\vec{z}_{\perp}-\vec{x}_{1 \perp}\right|^{2}} \cdot \frac{\vec{x}_{2 \perp}-\vec{b}_{2 \perp}}{\left|\vec{x}_{2 \perp}-\vec{b}_{2 \perp}\right|^{2}}+\frac{\vec{\varepsilon}_{\perp}^{\lambda *} \cdot\left(\vec{x}_{2 \perp}-\vec{b}_{2 \perp}\right)}{\left|\vec{x}_{2 \perp}-\vec{b}_{2 \perp}\right|^{2}} \frac{\vec{x}_{1 \perp}-\vec{b}_{2 \perp}}{\left|\vec{x}_{1 \perp}-\vec{b}_{2 \perp}\right|^{2}} \cdot \frac{\vec{z}_{\perp}-\vec{x}_{2 \perp}}{\left|\vec{z}_{\perp}-\vec{x}_{2 \perp}\right|^{2}}\right] \\
& \times f^{a b c}\left[U_{\vec{x}_{1 \perp}}^{b d}-U_{\vec{b}_{2 \perp}}^{b d}\right]\left[U_{\vec{x}_{2 \perp}}^{c e}-U_{\vec{b}_{2 \perp}}^{c e}\right]\left(V_{\vec{b}_{1 \perp}}\right)_{1}\left(V_{\vec{b}_{2 \perp}} t^{e} t^{d}\right)_{2} \\
& +\frac{i g^{3}}{4 \pi^{3}} \int d^{2} x f^{a b c} U_{\vec{b}_{2 \perp}}^{b d}\left[U_{\vec{x}_{\perp}}^{c e}-U_{\vec{b}_{2 \perp}}^{c e}\right]\left(V_{\vec{b}_{1 \perp}}\right)_{1}\left(V_{\vec{b}_{2 \perp}} t^{e} t^{d}\right)_{2}\left(\frac{\vec{\varepsilon}_{\perp}^{\lambda *} \cdot\left(\vec{z}_{\perp}-\vec{x}_{\perp}\right)}{\left|\vec{z}_{\perp}-\vec{x}_{\perp}\right|^{2}} \frac{1}{\left|\vec{x}_{\perp}-\vec{b}_{2 \perp}\right|^{2}}\right. \\
& \left.-\frac{\vec{\varepsilon}_{\perp}^{\lambda *} \cdot\left(\vec{z}_{\perp}-\vec{b}_{2 \perp}\right)}{\left|\vec{z}_{\perp}-\vec{b}_{2 \perp}\right|^{2}} \frac{\vec{z}_{\perp}-\vec{x}_{\perp}}{\left|\vec{z}_{\perp}-\vec{x}_{\perp}\right|^{2}} \cdot \frac{\vec{x}_{\perp}-\vec{b}_{2 \perp}}{\left|\vec{x}_{\perp}-\vec{b}_{2 \perp}\right|^{2}}-\frac{\vec{\varepsilon}_{\perp}^{\lambda *} \cdot\left(\vec{z}_{\perp}-\vec{b}_{2 \perp}\right)}{\left|\vec{z}_{\perp}-\vec{b}_{2 \perp}\right|^{2}} \frac{1}{\left|\vec{x}_{\perp}-\vec{b}_{2 \perp}\right|^{2}}\right) \\
& +\frac{i g^{3}}{4 \pi^{2}} f^{a b c} U_{\vec{b}_{2 \perp}}^{b d}\left[U_{\vec{z}_{\perp}}^{c e}-U_{\vec{b}_{2 \perp}}^{c e}\right]\left(V_{\vec{b}_{1 \perp}}\right)_{1}\left(V_{\vec{b}_{2 \perp}} t^{e} t^{d}\right)_{2} \frac{\vec{\varepsilon}_{\perp}^{\lambda *} \cdot\left(\vec{z}_{\perp}-\vec{b}_{2 \perp}\right)}{\left|\vec{z}_{\perp}-\vec{b}_{2 \perp}\right|^{2}} \ln \frac{1}{\left|\vec{z}_{\perp}-\vec{b}_{2 \perp}\right| \Lambda} .
\end{aligned}
$$

The E-diagrams term can be derived from Eq. 3.3 by simply switching the valance quarks.

\section{Conclusions and Outlook}

This is a serious attempt at analytically calculating the single inclusive gluon production crosssection for heavy-light ion collisions at the classical level, beyond the known leading order pA result derived in 1997 [3]. We have shown that all of the possible diagrams reduce to a few classical field diagrams with a single produced gluon in the amplitude. This resulted in a compact result in transverse coordinate space for the order- $g^{3}$ amplitude. Similar results in this direction have been obtained in [4], though due to apparent complexity of the later we were unable to perform a direct comparison with our results quoted earlier.

In order to calculate the single inclusive gluon production cross-section it is not enough to have the order $-g^{3}$ amplitude, we need the order $-g^{5}$ amplitude as well (see Fig. 2 (ii)). This is currently a work in progress.

\section{References}

[1] G. A. Chirilli, Y. V. Kovchegov, D. E. Wertepny, Classical Gluon Production Amplitude for Nucleus-Nucleus Collisions: First Saturation Correction in the Projectile, JHEP 1513 (2015) 015 [arxiv:1501.03106].

[2] L. D. McLerran and R. Venugopalan, Green's functions in the color field of a large nucleus, Phys. Rev. D50 (1994) 2225-2233 [hep-ph/ 9402335$].$

[3] Y. V. Kovchegov and A. H. Mueller, Gluon production in current nucleus and nucleon nucleus collisions in a quasi-classical approximation, Nucl. Phys. B529 (1998) 451-479 [hep-ph/9802440].

[4] I. Balitsky, Scattering of shock waves in QCD, Phys. Rev. D70 (2004) 114030 [hep-ph/ 0409314 ]. 